\title{
WILEY-VCH
}

\section{Elucidating and Mitigating Degradation Processes in Perovskite Light-Emitting Diodes}

Zahra Andaji-Garmaroudi, Mojtaba Abdi-Jalebi, Felix U. Kosasih, Tiarnan Doherty, Stuart Macpherson, Alan R. Bowman, Gabriel J. Man, Ute B. Cappel, Håkan Rensmo, Caterina Ducati, Richard H. Friend, and Samuel D. Stranks*

Z. Andaji-Garmaroudi, Dr. Mojtaba Abdi-Jalebi, T. Doherty, S. Macpherson, A. R. Bowman, Prof. R. H. Friend, Dr. S. D. Stranks

Cavendish Laboratory, Department of Physics, University of Cambridge, JJ Thomson

Avenue, Cambridge CB3 0HE, United Kingdom

E-mail:sds65@cam.ac.uk

Dr. M. Abdi-Jalebi

Institute for Materials Discovery, University College London, Torrington Place, London WC1E 7JE, United Kingdom

F. U. Kosasih, Prof. C. Ducati

Department of Materials Science and Metallurgy, University of Cambridge, 27 Charles Babbage Road, Cambridge CB3 0FS, United Kingdom

Dr. G. J. Man, Dr. U. B. Cappel, Prof. H. Rensmo

Division of Molecular and Condensed Matter Physics, Department of Physics and Astronomy, Uppsala University, Box 516, 75120 Uppsala, Sweden

Dr. S. D. Stranks

Department of Chemical Engineering and Biotechnology, University of Cambridge, Philippa Fawcett Drive, Cambridge CB3 0AS, United Kingdom

Keywords: halide perovskites; light-emitting diodes; degradation; ion migration; passivation 


\section{WILEY-VCH}

\section{Abstract}

Halide perovskites have attracted substantial interest for their potential as disruptive display and lighting technologies. However, perovskite light-emitting diodes (PeLEDs) are still hindered by poor operational stability. A fundamental understanding of the degradation processes is lacking but will be key to mitigating these pathways. Here, we use a combination of in operando and ex-situ measurements to monitor the performance degradation of $\left(\mathrm{Cs}_{0.06} \mathrm{FA}_{0.79} \mathrm{MA}_{0.15}\right) \mathrm{Pb}\left(\mathrm{I}_{0.85} \mathrm{Br}_{0.15}\right)_{3}$ PeLEDs over time. Through device, nanoscale crosssectional chemical mapping, and optical spectroscopy measurements, we reveal that the degraded performance arises from an irreversible accumulation of bromide content at one interface, which leads to barriers to injection of charge carriers and thus increased non-radiative recombination. We impede this ionic segregation by passivating the perovskite films with potassium halides, which immobilizes the excess halide species. The passivated PeLEDs show enhanced external quantum efficiency (EQE) from 0.5 to $4.5 \%$ and, importantly, show significantly enhanced stability, with minimal performance roll-off even at high current densities $\left(>200 \mathrm{~mA} / \mathrm{cm}^{2}\right)$. The decay half-life for the devices under continuous operation at peak EQE increases from $<1$ hour to $\sim 15$ hours through passivation, and $\sim 200$ hours under pulsed operation. Our results provide generalized insight into degradation pathways in PeLEDs and highlight routes to overcome these challenges. 


\section{WILEY-VCH}

\section{Main Text}

Metal halide perovskites with a formula of $\mathrm{ABX}_{3}$, where A represents a monovalent cation, $\mathrm{B}$ is a divalent metal cation, and $\mathrm{X}$ represents a halide anion, have been widely used in solar cells, lasers, detectors and light-emitting diodes (LEDs) owing to their exceptional optical and electronic properties such as long charge-carrier diffusion lengths, high absorption coefficients, tunable bandgaps, and facile processing. ${ }^{[1-4]}$ For bulk polycrystalline perovskite thin films, one of the key approaches to boost the stability and optoelectronic properties is through employing mixtures of A-site cations such as Methylammonium (MA), Formamidinium (FA), and Cesium $(\mathrm{Cs})^{[5,6]}$ as well as mixtures of $\mathrm{X}$-site anions such as Bromide $(\mathrm{Br})$ and Iodide $(\mathrm{I}){ }^{[7,8]}$

Recently, rapid progress has been made in improving the performances of perovskite lightemitting diodes (PeLEDs), ${ }^{[9-14]}$ yet their practical commercial implementation is still severely hindered by poor operational lifetimes. ${ }^{[10,15-19]}$ Although the sensitivity of the devices to atmospheric effects such as oxygen and water can be mitigated through effective packaging techniques, ${ }^{[20]}$ there is also an intrinsic instability in the devices with the high electric fields experienced under LED operation. ${ }^{[21]}$ This instability appears to be caused by a combination of ionic migration and Joule heating, ${ }^{[20,22-26]}$ which ultimately leads to degradation of the device performance. In order to overcome these degradation processes, a comprehensive understanding of the degradation location in the device and underlying degradation mechanisms under device operation conditions is essential, thereby allowing a rational approach to stabilize operation.

Here, we fabricate triple-cation mixed-halide $\left(\mathrm{Cs}_{0.06} \mathrm{FA}_{0.79} \mathrm{MA}_{0.15}\right) \mathrm{Pb}\left(\mathrm{I}_{0.85} \mathrm{Br}_{0.15}\right)_{3} \mathrm{PeLEDs}$ and monitor in operando the degradation of device performance over time. We observe a substantial drop in the external quantum efficiency (EQE) to negligible values accompanied by a red-shift of the electroluminescence (EL) over time under operation. We use a combination of in- and ex-situ device, nanoscale cross-sectional chemical mapping, and optical spectroscopy measurements to show that the degraded performance is caused by accumulation of bromide 


\section{WILEY-VCH}

content at the bottom interface with the electron-injecting layer, leading to increased nonradiative recombination. Through use of potassium halide salts, we demonstrate that the segregation effects can be mitigated, leading to increased EQE of the devices from $0.5 \%$ to $4.5 \%$ and substantially enhanced stability, with minimal roll-off of performance even at high current densities $\left(>200 \mathrm{~mA} / \mathrm{cm}^{2}\right)$ and an order-of-magnitude increase in the half-life decay from the peak EQE value under continuous operation to $\sim 10$ hours. Our results elucidate degradation pathways in PeLEDs and demonstrate strategies to overcome such critical hurdles to commercialisation.

We fabricated LED devices with the architecture indium-tin oxide (ITO) / bilayer of aluminium doped zinc oxide (AZO) and poly-ethylenimine (PEIE) ( $\sim 30 \mathrm{~nm}$ total thickness) / perovskite ( 200 nm) / poly(9,9-dioctyl-fluorene-co- $N$-(4-butylphenyl)diphenylamine) (TFB) $(\sim 50 \mathrm{~nm}) / \mathrm{MoO}_{3}(\sim 8 \mathrm{~nm}) / \mathrm{Au}(\sim 100 \mathrm{~nm})$ (see schematic in Figure 1a inset, and experimental section for details). Here, the perovskite layer is a $\left(\mathrm{Cs}_{0.06} \mathrm{FA}_{0.79} \mathrm{MA}_{0.15}\right) \mathrm{Pb}\left(\mathrm{I}_{0.85} \mathrm{Br}_{0.15}\right)_{3}$ perovskite thin film solution-processed from a precursor solution comprised of relevant salts dissolved in a mixture of $N, N$-dimethylformamide (DMF) and dimethyl sulfoxide (DMSO). The workhorse triple cation perovskite makes for an excellent demonstrator system to draw general conclusions around the degradation and ion migration processes in PeLEDs. Upon application of forward bias (2 V), we observe a sharp EL spectrum with peak at $\sim 765 \mathrm{~nm}$ (Figure 1a, black spectrum). We record the EL spectra over time under fixed operation at $2 \mathrm{~V}$ (Figure 1a), observing that the EL peak initially increases in magnitude over the first 10 minutes, before then quickly decreasing in magnitude over the course of the following 3 hours, concomitant with a red-shift of the spectrum. We show in Figure $1 \mathrm{~b}$ the radiance, current density, and relative EQE (ratio of radiance to current density) of devices, each normalized to their respective peak values, under continued operation over 10 hours held at fixed bias of $2 \mathrm{~V}$. We observe that over the first 15 minutes, both the current and radiance (and therefore relative EQE) increase to peak values, with the current peaking slightly earlier in time than the radiance (Figure 1b, inset). We attribute 


\section{WILEY-VCH}

these observations to a luminance overshoot arising from interfacial ion accumulation ${ }^{[26]}$ that may reduce defect densities ${ }^{[27]}$ or better balance charge injection; this slow burn-in process appears to be analogous to light-induced luminescence increases reported in neat films and solar cells. ${ }^{[28]}$ After this peak, the current decreases slowly, with a half-life from the peak of $\sim 3.5$ hours. However, the radiance, and hence relative EQE, decreases very rapidly after the peak value, with a half-life of only 55 minutes. This decay of device radiance under long-term operation does not recover even when the device is switched off for extended periods (Figure $\mathrm{S} 1)$.

To further probe these effects, we now study a pristine device (termed 'Pristine' herein) that hasn't been operated and a device that has been continuously operated for 3 hours at a fixed bias of $2 \mathrm{~V}$ corresponding to the vertical dashed line in Figure $1 \mathrm{~b}$ (termed 'Operated'). We reveal an increased radiance turn-on voltage from $\sim 1 \mathrm{~V}$ in the pristine device to $\sim 2 \mathrm{~V}$ after operation (Figure 1c), along with a shoulder in the current characteristics in the operated device at $\sim 2 \mathrm{~V}$ (Figure 1d; see Figure S2 for EQE-voltage plots); the origin of both effects will be discussed later. We also observe a clear increase in leakage current, reduction in radiance in the operated device across the measured voltage range and a reduced maximum radiance value from $1.71 \mathrm{WSr}^{-1} \mathrm{~m}^{-2}$ to $0.09 \mathrm{WSr}^{-1} \mathrm{~m}^{-2}$ (Figure $1 \mathrm{c}$ ). This reduction in radiance corresponds to reduced values for both photoluminescence lifetime and total integrated luminescence intensity of the perovskite in the operated device (Figure 1e). These results are consistent with suboptimal diode characteristics and increased non-radiative recombination in the degraded devices, which rationalizes the sharp drop in radiance (and hence EQE) of the device over time under operation. 

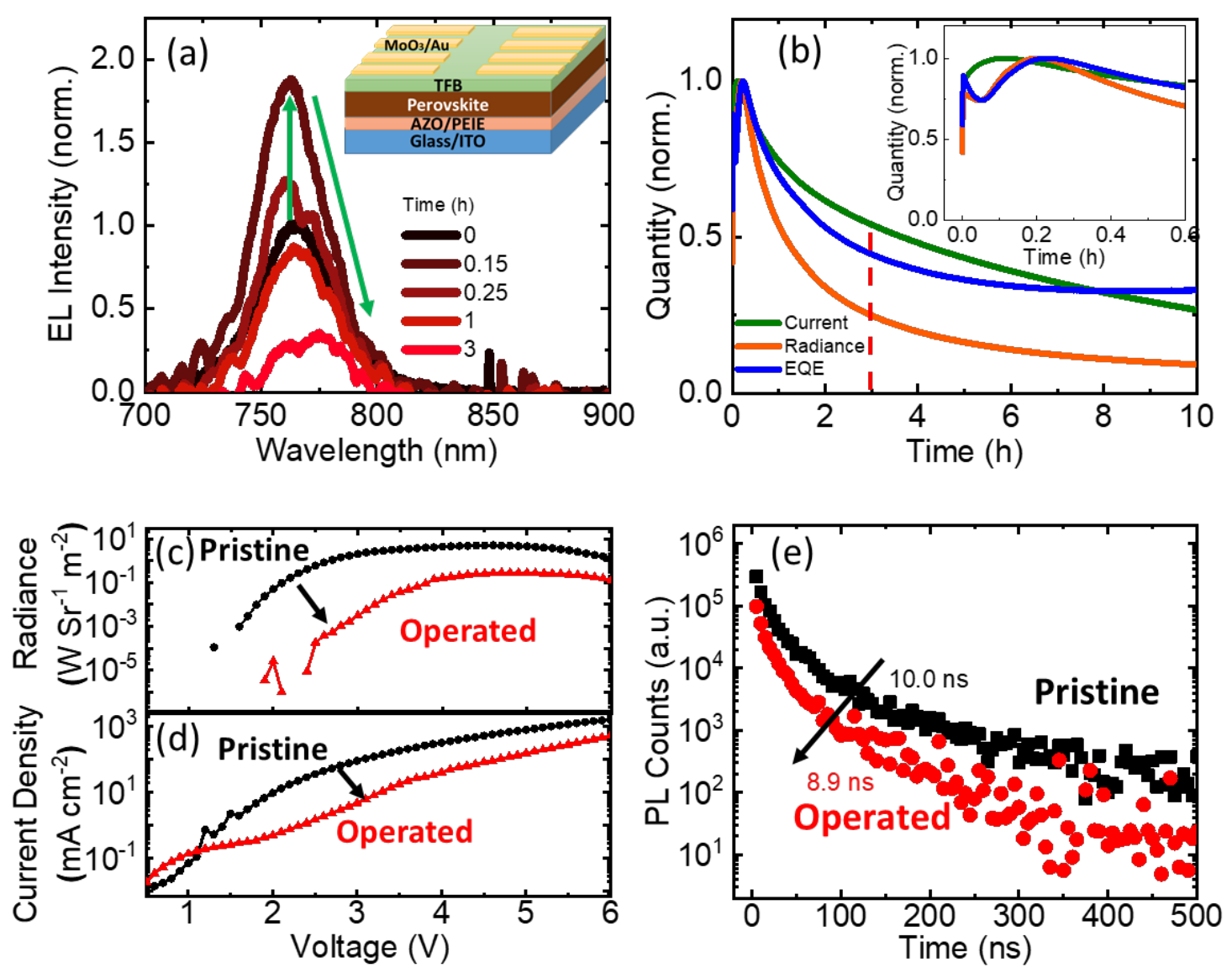

Figure 1. (a) EL spectra from a $\left(\mathrm{Cs}_{0.06} \mathrm{FA}_{0.79} \mathrm{MA}_{0.15}\right) \mathrm{Pb}\left(\mathrm{I}_{0.85} \mathrm{Br}_{0.15}\right)_{3}$ perovskite device (architecture shown in inset) over a period of 3 hours under operation at a constant forward bias voltage of $2 \mathrm{~V}$. (b) Current, radiance and EQE (the ratio of radiance to current) quantities of the device operated over a period of 10 hours at a fixed forward bias of $2 \mathrm{~V}$. Each quantity is normalized to its peak value. The inset shows the early time behaviour. (c) Radiance-voltage and (d) current density-voltage characteristics of a pristine device before operation (black) compared to another device that has been operated for 3 hours at a constant bias of $2 \mathrm{~V}$ (dashed line in b). (e) Time-resolved photoluminescence decays of the perovskite emission in the device without operation (pristine) compared to a device after operation at $2 \mathrm{~V}$ for 3 hours. Samples were excited with a $550-\mathrm{nm}$ pulsed source (pulse fluence of $1.8 \mu \mathrm{J} \mathrm{cm}^{-2}$, repetition rate of 1 $\mathrm{kHz}$ ). Stated lifetimes correspond to the time taken to fall to $1 / \mathrm{e}$ of the initial intensity.

To ascertain the origin of the decrease in luminescence efficiency after operation, we used scanning transmission electron microscopy imaging and energy-dispersive X-ray spectroscopy (STEM-EDX) to map the cross-sectional chemical composition of a pristine device (Figure 2a and d) and a companion device from the same batch operated for 3 hours at $2 \mathrm{~V}$ (Figure $2 \mathrm{~b}$ and e) (see experimental section for cross-sectional TEM sample preparation, and Figure S3 for 


\section{WILEY-VCH}

STEM-EDX maps across larger areas of the device). We observe that after operation there is a distinct depletion of bromide in the upper region of the film, which instead accumulates close to the bottom AZO/PEIE electron-injecting layer. We note that we do not observe this accumulation in pristine LEDs or solar cells utilising these compositions. ${ }^{[29]}$ This effect can also be seen when comparing the integrated line profiles of regions without voids or pinholes in Figure 2c (see Figure S3 for the regions that have been integrated over for line profile curves). In the opposite direction, we see that the homogeneous iodide distribution (Figure $2 \mathrm{~d}$ ) shows a small but broad shift towards the top perovskite/TFB interface after operation (Figure 2e and f). By contrast, the perovskite layer itself remains spatially homogeneous before and after operation (see Figure S3 and S4 for STEM-EDX results for all components and contact layers). Accumulation of negatively charged bromide ions on the electron injecting layer (cathode) interface shows that this movement cannot be explained by considering external electric fields. We speculate that the ionic migration is induced by charge carrier gradients under operation, which may arise from accumulation of electrons on the top surface due to deep hole traps in that region ${ }^{[30]}$ and/or unbalanced charge injection processes with the typical charge injecting layers employed. In this case, field-induced ion migration would drive the more mobile bromide species $^{[31]}$ to the bottom interface; indeed, accumulation of ions at the cathode has been reported in other systems with similar vertical device architectures ${ }^{[32]}$. Heating effects and the unique phase behaviour of bromide/iodide mixtures may play additional roles in the segregation. 

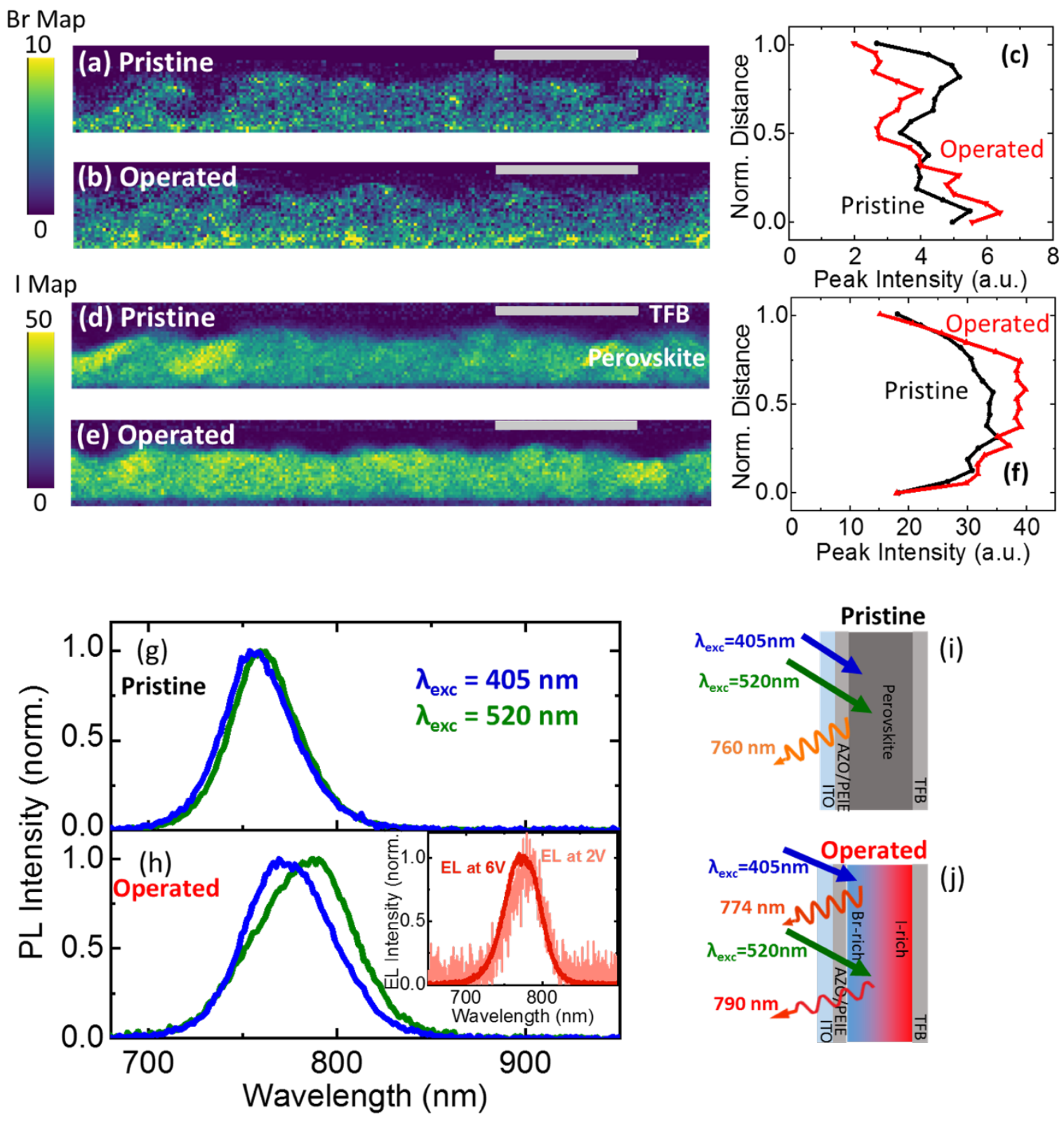

Figure 2. STEM-EDX maps of $(\mathrm{a}, \mathrm{b})$ bromine $\left(\mathrm{Br}-\mathrm{K}_{\alpha}\right)$, and $(\mathrm{d}, \mathrm{e})$ iodine $\left(\mathrm{I}-\mathrm{L}_{\alpha}\right)$ distributions for a pristine device (a,d), and a device after 3 hours of operation at $2 \mathrm{~V}(\mathrm{~b}, \mathrm{e})$. All scale bars represent $500 \mathrm{~nm}$. Panels (c) and (f) show the averaged intensities for bromine and iodine, respectively, through the normalized thickness of the films (from 0 , bottom, to 1 , top of film) in the regions highlighted in Figure S3. PL spectra from the (g) pristine device, and $(\mathrm{h})$ degraded device (Operated) with excitation at $\lambda_{\text {exc }}=405 \mathrm{~nm}$ (blue) and $\lambda_{\text {exc }}=520 \mathrm{~nm}$ (green) each at an excitation density of $\sim 60 \mathrm{~mW} / \mathrm{cm}^{2}$. Excitation and detection are on the bottom (glass) side of the device in both cases. The inset of (h) shows the EL spectra of the degraded device at a bias of $2 \mathrm{~V}$ and $6 \mathrm{~V}$. Schematic diagrams of the PL measurements for the (i) pristine, and (j) operated device, with arrows denoting excitation (right-moving) and emission (left-moving) photons.

To connect the halide segregation results to the PeLED optoelectronic properties, we further analyze the PL properties of a pristine device and a device continuously biased at $2 \mathrm{~V}$ for 3 


\section{WILEY-VCH}

hours. Here, we photo-excite the sample and monitor the emission from the bottom glass side of the device, in line with the direction of light emission from the PeLED and on the side of the interfacial bromide accumulation. For the pristine device before operation, we observe that excitation with either a 405-nm or 520-nm source results in relatively similar PL spectra with peak at $\sim 760 \mathrm{~nm}$ (Figure $2 \mathrm{~g}$ ), consistent with a perovskite layer with well intermixed halide components throughout the film thickness (Figure 2i). However, when we perform the same measurement on the operated (degraded) device, we see a red-shift and broadening of peaks from both excitation wavelengths (Figure 2h). Specifically, the PL peak when excited at 405 $\mathrm{nm}$ shifts to $\sim 774 \mathrm{~nm}$ with a broad lower energy tail, while the PL peak when excited at 520 $\mathrm{nm}$ shifts further to $\sim 790 \mathrm{~nm}$, with a significant shoulder at $\sim 775 \mathrm{~nm}$. We explain these observations by considering the halide segregation observed in Figure $2 \mathrm{c}$ and $2 \mathrm{f}$ for the operated devices in which the perovskite is more bromide-rich (larger bandgap) on the bottom part of the film, and more iodide-rich (lower bandgap) further into the bulk of the film. Excitation with 405-nm photons will primarily excite the bromide-rich bottom part (absorption depth of $\sim 25$ $\mathrm{nm}$ ), while 520-nm photons penetrate further into the bulk of the film (absorption depth of $\sim 70$ $\mathrm{nm})$. Thus, excitation with 520-nm photons will yield a greater fraction of lower energy, iodiderich bulk emission ( $790 \mathrm{~nm}$ ) than 405-nm excitation (Figure 2j). Furthermore, 520-nm photons will not be as significantly absorbed as $405-\mathrm{nm}$ photons by any perovskite material with very large fractions of bromide, components of which may well reside on the bottom interface, again leading to emission primarily from the lower bandgap iodide-rich components at this excitation wavelength. PL peaks for both excitation wavelengths in the operated device (Figure $2 \mathrm{~h}$ ) are red-shifted with respect to the pristine devices (Figure $2 \mathrm{~g}$ ) because of additional energy transfer of excitations down a gradient of bromide-to-iodide rich domains to these lower bandgap iodide-rich sites, ${ }^{[33]}$ though this process is not efficient enough for all excitations to transfer in this way. We note that the PL peak differences between the two excitation wavelengths is even more exaggerated at higher excitation fluences (Figure S5), which is particularly evident by the 


\section{WILEY-VCH}

PL peak with 405 -nm excitation now being blue-shifted to $\sim 750 \mathrm{~nm}$, at even higher energy than the pristine device $(\sim 760 \mathrm{~nm})$. This observation is also consistent with a small blue shift in the EL spectra of the operated device at $6 \mathrm{~V}$ (with higher carrier densities) compared to at $2 \mathrm{~V}$ (Figure $2 \mathrm{~h}$, inset). These results at higher carrier densities are consistent with radiative recombination on the bromide-rich layers being able to more effectively compete with processes including downhill energy transfer, charge trapping, and charge quenching by the adjacent contact compared to measurements at lower carrier densities. ${ }^{[33]}$

We conclude from these collective results that this large bandgap material (and any other degradation products) on the bottom interface will lead to important charge-carrier injection and quenching issues at that interface, resulting in increased non-radiative recombination. In fact, we observe low radiance values at $2 \mathrm{~V}$ after the operation period (cf. Figure 1c), suggesting that this bias is not large enough to inject sufficient carriers; only at higher bias do we recover some modest radiance, when the bias is high enough to inject carriers to achieve detectable EL signal (Figure 1c). This result is also supported by the current-voltage plots, which show a knee at $2 \mathrm{~V}$ and increased current above this bias value (Figure 1d). Furthermore, the reduced PL lifetime and intensity in the degraded device reported in Figure 1e is consistent with additional charge quenching and/or trap-related recombination; the segregated ions and more disordered perovskite likely lead to non-radiative recombination sites near the contact interface and/or undergo further chemical reactions with the transport layers or electrodes to generate interfacial defects or phase impurities. ${ }^{[22,34,35]}$ These assertions of increased non-radiative recombination are also consistent with the peak radiance in the degraded device being much lower than in the pristine device (cf. Figure 1c). The degraded interface and segregated emitter material will also lead to increased shunt pathways, explaining the increased leakage current (cf. Figure 1c). Our combined spectroscopic, device and chemical mapping results reveal that the halide segregation leads to increased non-radiative recombination in the operated device owing to a combination of carrier injection barriers leading to a potentially more unbalanced population of electron and 


\section{WILEY-VCH}

hole carriers, carrier trapping, and charge quenching. We note that the current and radiance onsets are both at $2 \mathrm{~V}$ (Figure $1 \mathrm{c}$ and $\mathrm{d}$ ), closely matching the magnitude of bias applied for the 3 hours of operation, suggesting that the ions arrange to balance internal fields; this observation is further supported by a comparable degradation measurement performed with a bias of $3 \mathrm{~V}$, in which the current and radiation onsets are also shifted to comparably higher voltage (Figure S6).

In order to mitigate the halide segregation during operation, we passivate the perovskite films with potassium halides. Passivation with alkali metals such as potassium have been previously shown to reduce non-radiative losses and ion migration in perovskite devices. ${ }^{[36-41]}$ We prepared a series of passivated perovskite thin films by addition of different amounts of potassium iodide solution (in the range $x=0-0.44$, where $x$ is the fraction of potassium out of total monovalent cations; $[\mathrm{K}] /([\mathrm{K}]+[\mathrm{MA}]+[\mathrm{FA}]+[\mathrm{Cs}]))$ to different iodide/bromide composition solutions to explore the series $\left(\mathrm{Cs}_{0.06} \mathrm{FA}_{0.79} \mathrm{MA}_{0.15}\right) \mathrm{Pb}\left(\mathrm{I}_{1-\mathrm{y}} \mathrm{Br}_{\mathrm{y}}\right)_{3}$, with $y=0-1$ being the fraction of bromide out of total halide (with corresponding PL emission peaks spanning from $560-826 \mathrm{~nm}$, see Figure S7). To assess the resilience of the resulting films to photoinduced ion migration, we monitored the PL spectra of the films before and after continuous illumination at $\sim 60 \mathrm{~mW} / \mathrm{cm}^{2}$ over a period of 30 minutes. We found that a sufficient fraction of added potassium is able to stabilize the bromide fractions $y<0.8$ against photo-induced ion migration under these test conditions, though there are some photo-induced changes seen for higher bromide fractions particularly at lower potassium fractions $(x<0.2)$.

We construct LED devices from a potassium series with nominal bromide fraction $y=0.15$ (corresponding to devices reported earlier for $x=0$, and the highest PL quantum efficiencies), and show EL spectra from the devices in Figure 3a. We observe a large red-shift (up to 40 $\mathrm{nm}$ ) of the emission with increasing potassium fraction, which cannot be ascribed solely to the small dilution of the total bromide content upon addition of KI through the recipe employed here. Rather, we attribute the red-shift to the potassium more selectively binding to the bromide 


\section{WILEY-VCH}

thus resulting in a more iodide-rich perovskite. ${ }^{[36]}$ This assertion is supported by soft and hard X-ray Photoelectron Spectroscopy (PES) measurements, which we use to differentiate between the halides bound to potassium versus the perovskite (Figure S8). Through use of appropriate $\mathrm{KBr}$ controls, we quantify the relative bromide fraction in the perovskite, compared to the amounts in $\mathrm{KBr}$, and plot this quantity for different probe depths in Figure 3c, where increasing probe photon energy corresponds to increasing depth from the surface (see Methods for details and Figure S9 for comparable iodide results). From this quantification, we find that the relative amount of bromide within the perovskite phase decreases by more than $50 \%$ upon addition of KI to the precursor solutions, and this relative amount increases with increasing probe depth for both samples (Figure 3c). Furthermore, a large fraction of the potassium salt is in the form of $\mathrm{KBr}$, explaining the decreased $\mathrm{Br}$ content within the perovskite phase, and resulting red-shifts of the EL peaks. The concentration of $\mathrm{Br}$ bound to $\mathrm{KBr}$ (and I bound to KI; Figure S9) appears depth-independent, suggesting that the $\mathrm{KI}$ and $\mathrm{KBr}$ crystallites are large in comparison to the PES probing depth (up to $\sim 30 \mathrm{~nm}$; further supported by STEM-EDX results below). Nevertheless, the total percentage of halide bound in potassium salts is approximately $25 \%$, i.e. higher than expected from synthesis (13\%), suggesting a larger concentration of $\mathrm{KI}$ and $\mathrm{KBr}$ at the perovskite surface than in the bulk. The addition of the potassium salt also increases the overall perovskite halide/lead ratio at the surface (Table S1), which is consistent with passivation through reduced surface halide vacancy concentration. ${ }^{[29]}$ 

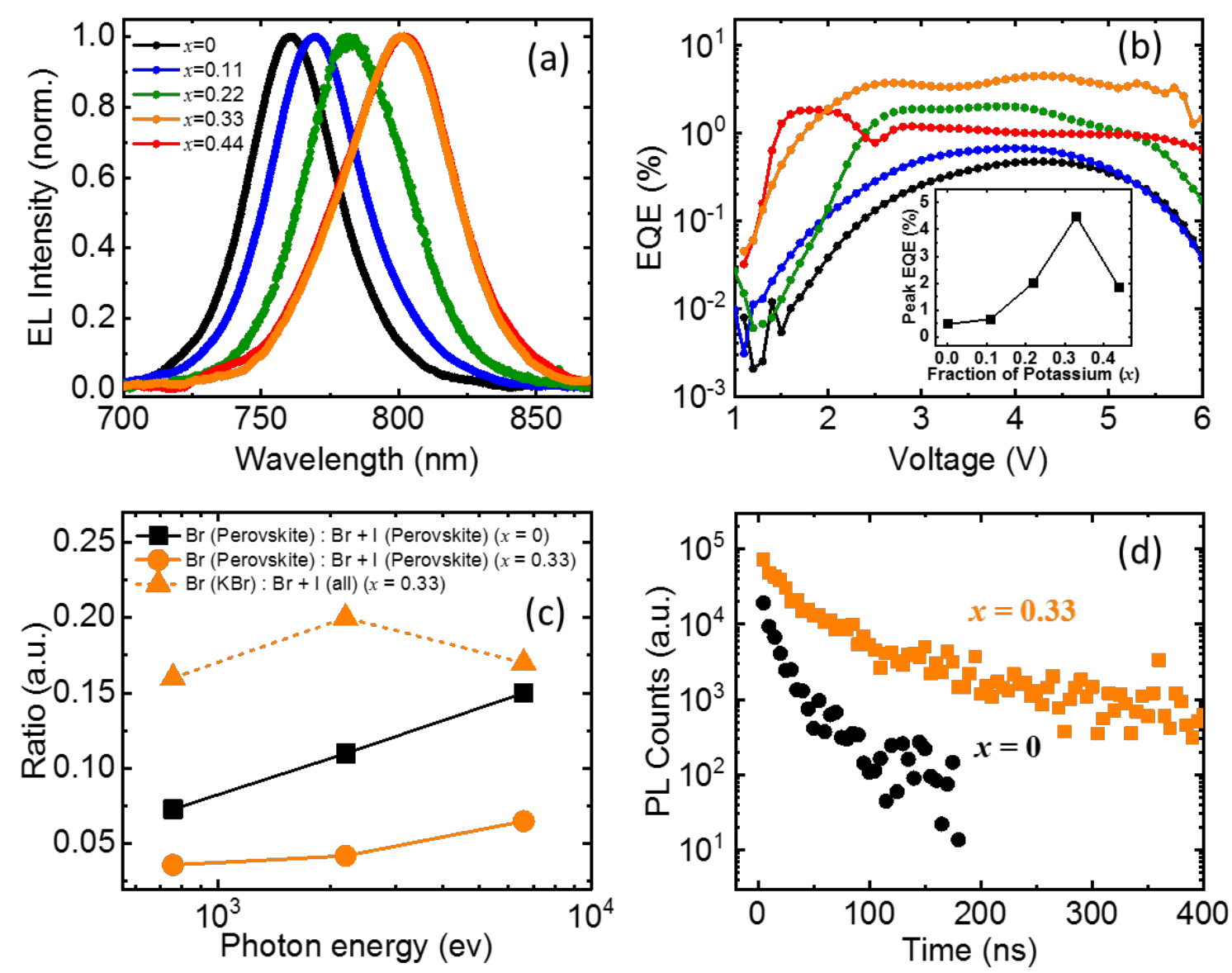

Figure 3. (a) Normalized EL spectra of the $\left(\mathrm{Cs}_{0.06} \mathrm{FA}_{0.79} \mathrm{MA}_{0.15}\right) \mathrm{Pb}\left(\mathrm{I}_{0.85} \mathrm{Br}_{0.15}\right)_{3}$ devices with different fractions of potassium $(x)$ as indicated, with bias at $2 \mathrm{~V}$. (b) EQE-voltage characteristics of devices with the same compositions, with peak EQE values shown in the inset. (c) Semi-log plots showing the Br-to-halide ratio specifically for the perovskite, and relative ratios of bromide attached to potassium bromide, as a function of photon energy (a proxy for probing depth), extracted from X-ray photoelectron spectroscopy (cf. Figure S8). (d) Timeresolved photoluminescence decays of the perovskite sandwiched between electron(AZO/PEIE) and hole-injecting (TFB/ $\mathrm{MoO}_{3}$ ) electrodes (i.e. stacks in the device but without ITO or $\mathrm{Au}$ ) for $x=0$ and $x=0.33$ compositions with excitation at $550 \mathrm{~nm}$ and a pulse fluence of $0.84 \mu \mathrm{J} \mathrm{cm}^{-2}$, repetition rate of $1 \mathrm{kHz}$.

The devices with the potassium additives show greatly enhanced EQE values (Figure 3b, inset) with a radiance onset as low as $\sim 1.1 \mathrm{~V}$ (Figure S10). The control device shows a peak EQE of $0.5 \%$, whereas the peak EQE of the best potassium-passivated device reaches $4.5 \%$ for $x=0.33$, which we study further herein (see Figure S10 for device statistics). Importantly, the high EQE value is stabilized across a wide voltage range with minimal roll-off even at high current densities (Figure 3b). For example, the EQE of the passivated $x=0.33$ device is $2.7 \%$ 


\section{WILEY-VCH}

even at $5.8 \mathrm{~V}$ (corresponding to $270 \mathrm{~mA} / \mathrm{cm}^{2}$ ), whereas the $\mathrm{EQE}$ of the control device under the same conditions is only $0.03 \%$ (corresponding to similar current density, $290 \mathrm{~mA} / \mathrm{cm}^{2}$ ). We attribute the enhanced EQE to an increased luminescence yield in the perovskite layer in the devices, as demonstrated by the increased PL lifetime at low excitation density (Figure 3d) for the perovskite sandwiched between the electron- and hole-injecting contacts for the passivated $x=0.33$ sample compared to the control $x=0$ (see Figure S11 for results on each individual contact). This is also consistent with the increased champion PL quantum efficiency (PLQE) of the perovskite in the full device stack from $0.5 \%(x=0)$ to $5 \%(x=0.33)$, with the reported EL EQE values at a comparable charge density consistent with what we would expect for these PLQE values. ${ }^{[42]}$

To compare the operational stability behaviour of the passivated devices to the control devices $(x=0)$ shown earlier, we continuously operated the $x=0.33$ passivated device and recorded the EL spectra over time at a fixed bias of $2 \mathrm{~V}$ (Figure 4a). We observe a similar but slower burn-in phenomenon to the unpassivated control sample in which the EL increases over time over the course of 3 hours (cf. Figure 1a) but, notably, the device is then stabilized and we do not see the subsequent reduction in EL intensity or shift in spectral shape that was seen in the control sample after just 10 minutes. We note that the absolute EQE at this peak is $6.5 \%$ (see Figure S12), increased from a value before operation of $4.5 \%$, though this increased EQE phenomenon is reversible when the bias is switched off (Figure S13). These observations are also reflected in the normalized time-series plots of radiance, current density and relative EQE of the passivated sample at constant $2 \mathrm{~V}$ bias shown in Figure $4 \mathrm{~b}$, in which the radiance reaches a peak after more than 3 hours and then shows a slow decay with a half-life of 10 hours (compared to 55 minutes for the control sample, see Figure $4 \mathrm{~b}$ inset for comparison). Similar behavior is observed under constant current conditions (Figure S13), with a decay half-life of 15 hours for the passivated sample at a fixed current corresponding to the peak EQE $\left(10 \mathrm{mAcm}^{-}\right.$

${ }^{2}$ ). We note that the current density also shows a prolonged decay trend (half-life of 11 hours 


\section{WILEY-VCH}

after reaching the peak value) compared to the control ( 2 hours) though this is not as stark as the increase in half-life of radiance. These stability improvements are evident even when comparing to an unpassivated $\left(\mathrm{Cs}_{0.06} \mathrm{FA}_{0.79} \mathrm{MA}_{0.15}\right) \mathrm{Pb}\left(\mathrm{I}_{0.95} \mathrm{Br}_{0.05}\right)_{3}$ control that has similar EL peak position and therefore similar incorporated bromide fraction to the $x=0.33$ $\left(\mathrm{Cs}_{0.06} \mathrm{FA}_{0.79} \mathrm{MA}_{0.15}\right) \mathrm{Pb}\left(\mathrm{I}_{0.85} \mathrm{Br}_{0.15}\right)_{3}$ device yet exhibits a rapid performance decay under operation (Figure S13), providing strong evidence that the stability enhancements are due to the potassium passivation rather than any small changes in bromide/iodide fraction.

To further demonstrate the stability of the passivated device, we show in Figure $4 \mathrm{c}$ the relative radiance from the device with a pulsed square wave bias comprised of a forward bias of $2 \mathrm{~V}(20 \%$ of cycle) and a reverse bias of $-0.5 \mathrm{~V}(80 \%$ of cycle), thus giving negligible net field across the device when time-integrated (see device parameters over the course of duty cycles in Figure S14, and Methods for further details). These data show that the rise to maximum radiance occurs over a period of 25 hours, with a subsequent half-life of the radiance of 200 hours; when considering the time under forward bias (and thus light out) the half-life is $\sim 40$ hours (Figure S15), a factor of four times longer than the devices under continuous operation ( $\sim 10$ hours). We explain these observations of impeded degradation by considering that the asymmetric carrier distributions we propose to be driving the residual ion migration under operation in these passivated devices are removed for extended periods between forward bias $(8 \mathrm{~ms})$ because carriers will recombine within microsecond time scales. 

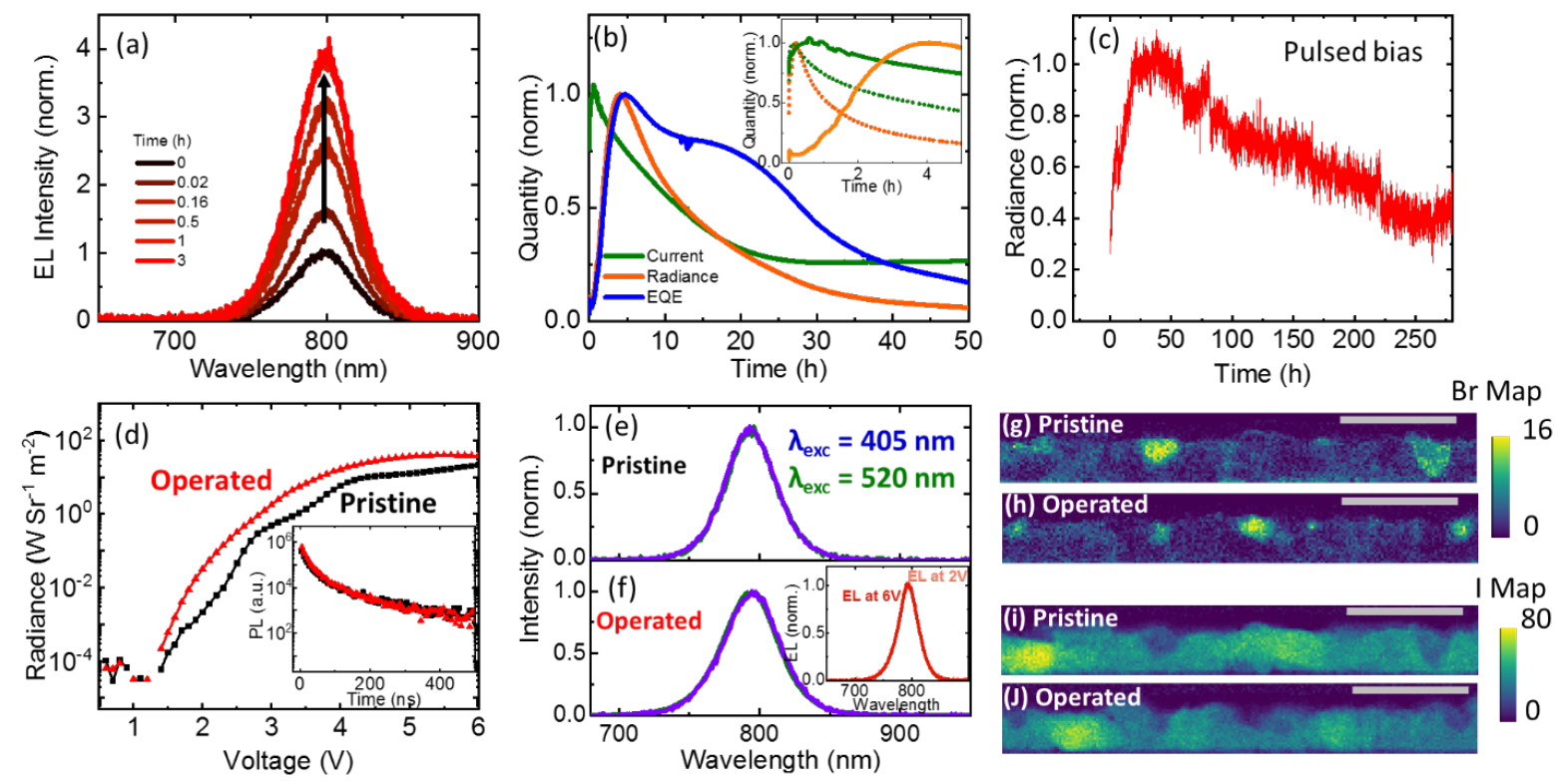

Figure 4. (a) EL spectra from a potassium-passivated $(x=0.33)$ $\left(\mathrm{Cs}_{0.06} \mathrm{FA}_{0.79} \mathrm{MA}_{0.15}\right) \mathrm{Pb}\left(\mathrm{I}_{0.85} \mathrm{Br}_{0.15}\right)_{3}$ perovskite device over a period of 3 hours under operation at a constant forward bias voltage of $2 \mathrm{~V}$. (b) Current, radiance and EQE (the ratio of radiance to current) quantities of the passivated device operated over a period of 50 hours at a fixed bias of $2 \mathrm{~V}$. Each quantity is normalized to its peak value. The inset shows the current and radiance over the early times for control ( $x=0$; dotted lines) and passivated ( $x=0.33$; solid lines $)$ devices. (c) Relative radiance intensity of the passivated device (normalized to the mean peak value) when biased with a square wave voltage profile at a forward bias of $2 \mathrm{~V}$ ( $20 \%$ of cycle) and a reverse bias of $-0.5 \mathrm{~V}$ ( $80 \%$ of cycle), giving no net time-integrated field. (d) Radiance-voltage characteristics of the passivated device before operation (black) compared to another passivated device after operation for 3 hours at a constant bias of $2 \mathrm{~V}$ (dashed line in b). Inset shows timeresolved photoluminescence decays of the perovskite emission in the full device without operation (Pristine) compared to a device after operation at $2 \mathrm{~V}$ for 2 hours (Operated). Samples were excited with a 550-nm pulsed source (pulse fluence of $1.8 \mu \mathrm{J} \mathrm{cm}{ }^{-2}$, repetition rate of 1 $\mathrm{kHz}$ ). PL spectra from (e) a pristine passivated device, and (f) a passivated device after operation for 3 hours at $2 \mathrm{~V}$ with excitation at $405 \mathrm{~nm}$ (blue) and $520 \mathrm{~nm}$ (green) at $\sim 60 \mathrm{~mW} / \mathrm{cm}^{2}$. Excitation and detection are on the bottom (glass) side of the device in both cases. The inset in $\mathrm{f}$ shows the EL spectra of the operated device at a bias of $2 \mathrm{~V}$ and $6 \mathrm{~V} .(\mathrm{g}, \mathrm{h})$ Bromine $\left(\mathrm{Br}-\mathrm{K}_{\alpha}\right)$, and $(\mathrm{i}, \mathrm{j})$ iodine $\left(\mathrm{I}-\mathrm{L}_{\alpha}\right)$ distributions for $(\mathrm{g}, \mathrm{i})$ a pristine passivated device, and $(\mathrm{h}, \mathrm{j})$ a passivated device after 3 hours of operation at $2 \mathrm{~V}$. All scale bars represent $500 \mathrm{~nm}$. Figure S16 show the averaged intensities for bromine and iodine through the depth of the films in the regions highlighted in the same figure.

To further understand the device behavior before and after operation for the passivated devices $(x=0.33)$, we show radiance-voltage plots for a pristine device and a device operated with continuous bias at $2 \mathrm{~V}$ for 3 hours in Figure $4 \mathrm{~d}$. We observe that the onset of radiance for the passivated device does not change after biasing, and in fact we observe an enhancement in the radiance across the full range of voltage, consistent with the increased radiance in Figure 


\section{WILEY-VCH}

4d. We observe similar small increases in the current, EQE (Figure S12), and integrated PL intensity under the PL decay (Figure $4 \mathrm{~d}$ inset). This is in sharp contrast to the control device ( $x$ $=0$ ) under the same operation conditions, which shows a dramatic drop in radiance (cf Figure 1c), current, EQE (Figure S1) and PL decay time (Figure 1d). We also do not see significant differences in the PL spectra with excitation at either $405 \mathrm{~nm}$ and $520 \mathrm{~nm}$ when comparing before (Figure 4e) and after (Figure 4f) device operation for the passivated samples, or changes in the EL spectra at $2 \mathrm{~V}$ or $6 \mathrm{~V}$ after operation (Figure $4 \mathrm{f}$ inset), consistent with an inhibition of ion segregation effects under operation. These observations are also consistent with STEMEDX cross-section results, which show very little change in the vertical distribution of the halides between a pristine and operate device, as shown in Figure $4 \mathrm{~g}$ and $\mathrm{h}$ (see Figure S16 for other compositional components, morphological images and line profiles). We note that fractions of the halides are associated with potassium halide inclusions, consistent with the PES results (Figure 3c), which are benign for device performance and immobilize further movement under operation. ${ }^{[36]}$

In both the passivated and unpassivated devices, there is an initial burn-in under operation which has been attributed to ion migration effects. ${ }^{[26]}$ However, the initial rate of the burn-in occurs on a faster time scale $\left(\sim 4 \mathrm{~h}^{-1}\right)$ in the control device compared to the passivated device $\left(\sim 0.4 \mathrm{~h}^{-1}\right)$. Furthermore, the enhancement in performance in the control device is quickly overwhelmed by a rapid degradation process and hence the relative increase in performance during the burn-in is limited. The addition of potassium salts slows the burn in process but, notably, significantly retards this degradation component, leading to a more prolonged burn-in period and much slower subsequent decay (by an order of magnitude). The bromide is critical in the film formation stage to ensure high film quality, ${ }^{[29]}$ but its subsequent presence poses a liability for stability; the potassium complexes with a large fraction of the bromide, which stabilizes the operation by inhibiting the accumulation of excess bromide on the bottom interface. The passivation of surface hole traps ${ }^{[30]}$ will also reduce unbalanced carrier 


\section{WILEY-VCH}

accumulation that may otherwise lead to field-induced ion segregation. This work thus provides a generalized template for further strategies for stabilizing PeLED performance, i.e. targeting the use of agents able to slow or even immobilize the movement of halide species within the emitter material, as well as passivate surfaces. This generalized template is consistent with other recent strategies successfully stabilizing PeLED operation including use of 2D molecules and $\mathrm{Cs} / \mathrm{Rb}$ components. ${ }^{[26,41]}$

In conclusion, using a combination of in- and ex-situ device, nano-scale chemical mapping cross-section, and optical spectroscopy measurements, we found that the reduction in the performance and luminescence yield of $\left(\mathrm{Cs}_{0.06} \mathrm{FA}_{0.79} \mathrm{MA}_{0.15}\right) \mathrm{Pb}\left(\mathrm{I}_{0.85} \mathrm{Br}_{0.15}\right)_{3}$ PeLED devices arises from an accumulation of bromide content at one interface and a more iodide-rich bulk material. This accumulation of ions leads to a defective perovskite layer that acts as an injection barrier for charge carriers and leads to increased non-radiative recombination. Our results show that a practical approach to improve the maximum EQE and stability of the devices is to retard the movement of halide species through halide sequestering species such as potassium salts. The potassium-passivated LEDs show a longer burn-in process and significantly prolonged decay of radiance and current density. Such strategies to inhibit halide segregation in the emitter material and at the interfaces will also prevent further chemical reactions with the contact layers and electrodes that will otherwise collectively limit PeLED commercialization opportunities. Our work thus provides a generalized template for seeking further strategies to stabilize PeLED operation.

\section{Experimental Section}

Materials: Lead compounds were purchased from TCI, the organic cations were purchased from Dyesol, and Cesium iodide and potassium iodide were obtained from Alfa Aesar. TFB was purchased from American Dye Source. Unless otherwise stated, all other materials were purchased from Sigma-Aldrich. 


\section{WILEY-VCH}

Film and device fabrication: Triple-cation mixed-halide perovskite $\left(\mathrm{Cs}_{0.06} \mathrm{FA}_{0.79} \mathrm{MA}_{0.15}\right) \mathrm{Pb}\left(\mathrm{I}_{0.85} \mathrm{Br}_{0.15}\right)_{3}$ precursor solution was prepared by dissolving $\mathrm{PbI}_{2}(1.10 \mathrm{M})$, $\mathrm{PbBr}_{2}(0.22 \mathrm{M})$, formamidinium iodide $(1.00 \mathrm{M})$, and methylammonium bromide $(0.20 \mathrm{M})$ in a mixture of anhydrous DMF:DMSO (4:1 volume ratio, v:v) followed by addition of 5 vol\% from CsI stock solution (1.5 M in DMSO). The potassium iodide was first dissolved in a mixed solution of DMF:DMSO 4:1 (v:v) to make a stock solution of $1.5 \mathrm{M}$. We then added the potassium iodide solution into the triple cation perovskite solution in different volume ratios between 0.1 to 0.4 . We then diluted the final solutions with different volume percentages of anhydrous DMF:DMSO (4:1 volume ratio, v:v) to reach $200 \mathrm{~nm}$ thickness for deposited films. We diluted the solutions by 66 vol\% for $x=0,61$ vol $\%$ for $x=0.11,55 \operatorname{vol} \%$ for $x=0.22,50$ $\operatorname{vol} \%$ for $x=0.33$, and $45 \operatorname{vol} \%$ for $x=0.44$. ITO and glass substrates were cleaned by sonication in acetone and isopropyl alcohol for $30 \mathrm{~min}$, then the substrates were further cleaned with oxygen plasma treatment for $10 \mathrm{~min}$. The perovskite solution was spin-coated in a two-step program at 1,000 and 4,000 rpm for 10 and $30 \mathrm{~s}$ respectively, and $110 \mu \mathrm{l}$ of chlorobenzene was cast on the spinning substrate $30 \mathrm{~s}$ after starting the program. The substrates were then annealed at $100^{\circ} \mathrm{C}$ for 30 minutes. Synthesis and deposition of perovskite solutions were performed inside a nitrogen glove box under moisture- and oxygen-controlled conditions $\left(\mathrm{H}_{2} \mathrm{O}\right.$ level: $<1$ ppm and $\mathrm{O}_{2}$ level: $<10 \mathrm{ppm}$ ). The thickness of the prepared films were $\sim 200 \mathrm{~nm}$. For the colloidal aluminium-doped zinc oxide in ethanol we spin coated on ITO at $4000 \mathrm{rpm}$ for 40 seconds to form the electron transport layer. A mixture of PEIE: 2-Methoxyethanol (1:100 weight ratio, w:w) was then spin coated on top of the AZO layer at $5000 \mathrm{rpm}$ for $60 \mathrm{~s}$. On top of the perovskite film, a TFB solution $(10 \mathrm{mg} / \mathrm{ml})$ was spin coated from toluene solution at 2000 rpm for $40 \mathrm{~s}$. Finally, $8 \mathrm{~nm}$ of MoOx and $100 \mathrm{~nm}$ of gold were sequentially evaporated as top electrodes through a shadow mask. All devices were encapsulated with UV epoxy (NOA81, 


\section{WILEY-VCH}

Thorlabs)/cover glass to avoid exposure to oxygen and moisture and degradation during measurement.

Device characterization: Current density-voltage $(\mathrm{J}-\mathrm{V})$ characteristics were measured using a Keithley 2400 source-meter unit. A silicon photodetector was placed at a large distance (40 $\mathrm{mm})$ compared to the dimensions of the emitting area $\left(4.5 \mathrm{~mm}^{2}\right)$ and the thickness of the device. The photon flux was measured simultaneously using this calibrated silicon photodiodes which operates in a photoamperic mode with a precision $1.0 \mathrm{M} \Omega$ gain transimpedance amplifier (sensitivity $H=6.2 \times 1012 \mathrm{e}^{\cdot} \mathrm{s}^{-1} \cdot \mathrm{V}^{-1}$ ). The radiance (in $\mathrm{W} \mathrm{sr}^{-1} \mathrm{~m}^{-2}$ ) of the devices was calculated based on the emission function of the PeLEDs and on the known spectral response of the silicon photodiode, and the EQEs were calculated assuming a Lambertian profile. ${ }^{[15]}$ The electroluminescence spectra of the devices were measured using a calibrated iCCD camera system (AndoriStar DH740 CCI-010) connected to a grating spectrometer (Andor SR303i).

Time-resolved photoluminescence measurements: Time-resolved PL spectra were recorded using a gated intensified CCD camera (Andor iStar DH740 CCI-010) connected to a calibrated grating spectrometer (Andor SR303i). A Ti:Sapphire optical amplifier (1 kHz repetition rate, 90 fs pulse width) was used to generate narrow bandwidth photoexcitations (10 nm FWHM) with a wavelength of $400 \mathrm{~nm}$ and $550 \mathrm{~nm}$, via a custom-built non-collinear optical parametric amplifier. Biasing of devices was performed during the measurement using a Keithley 2400 source-meter unit. The emitted steady state photoluminescence was collected via an optical fiber coupled to an Ocean Optics Maya2000 Pro spectrometer.

Photoluminescence quantum efficiency characterization: Fluorescence spectra and the external PL quantum efficiency was measured using an integrating sphere and a 532-nm continuous-wave laser at an illumination intensity of $\sim 60 \mathrm{~mW} / \mathrm{cm}^{2}$. We used a calibrated Andor silicon detector to determine photoluminescence quantum efficiency. The external PLQE was calculated as described elsewhere. ${ }^{[43]}$ 


\section{WILEY-VCH}

Scanning transmission electron microscopy-energy dispersive $X$-ray spectroscopy: Crosssectional TEM sample lamellae were cut and thinned to electron transparency with an FEI Helios Nanolab Dualbeam FIB/SEM following a standard protocol. ${ }^{[44]}$ The lamellae were then directly transferred into an FEI Osiris TEM operated at $200 \mathrm{kV}$, minimizing air exposure to about 2 minutes. STEM images were acquired in high-angle annular dark field (HAADF) mode using a Fischione detector with a dwell time of $1 \mu \mathrm{s} /$ pixel. STEM-EDX spectrum images were acquired using a Bruker Super-X silicon drift detector with a $\sim 0.9$ sr collection solid angle. To minimize beam damage, we used a defocused beam $(\Delta \mathrm{f}=-0.5 \mu \mathrm{m})$ with a beam current of $\sim 250 \mathrm{pA}$, dwell time of $40 \mathrm{~ms} /$ pixel, and spatial sampling of $10 \mathrm{~nm} / \mathrm{pixel}$. STEM-EDX data was denoised with principal component analysis (PCA) in HyperSpy. For detailed information on the application of PCA for STEM-EDX data, see ref. ${ }^{[45]}$

Soft and hard X-ray photoelectron spectroscopy: Photoelectron spectra were recorded from polycrystalline thin films, prepared in the same manner as full devices but without the TFB, $\mathrm{MoO}_{3}$ and $\mathrm{Au}$ overlayers, at beamline $\mathrm{I} 09$ at the Diamond Light Source (DLS, Oxfordshire, UK). To minimize the exposure of the films to air, the samples were transported from Cambridge to Diamond in bags filled with inert gas, mounted onto sample plates inside a glovebox at Diamond, and transported to the load lock in a suitcase. For each sample, photoelectron spectroscopy (PES) measurements were performed at room temperature with one soft X-ray photon energy $(758 \mathrm{eV})$ and two hard X-ray photon energies $(2200$ and $6600 \mathrm{eV})$ for depth-profiling purposes. Using the software QUASES, which calculates the inelastic mean free path (IMFP) using the TPP-2M formula, ${ }^{[46]}$ the probing depths (defined as three IMFP lengths) were calculated to be $\sim 5 \mathrm{~nm}(758 \mathrm{eV}), \sim 13 \mathrm{~nm}(2.2 \mathrm{keV})$ and $\sim 32 \mathrm{~nm}(6.6 \mathrm{keV})$,

respectively, by using $\mathrm{PbI}_{2}$ as a proxy for the perovskite compounds and electron kinetic energies corresponding to electrons photo-emitted from shallow core levels (e.g. Pb5d, I4d, Br3d). The Scienta EW4000 electron spectrometer was configured for pass energies of $100 \mathrm{eV}$ (758 eV), $200 \mathrm{eV}(758,2200,6600 \mathrm{eV})$ and $500 \mathrm{eV}(6600 \mathrm{eV})$. No charge neutralization was 


\section{WILEY-VCH}

used. Beam-induced sample damage was monitored by recording several iterations of the same core level spectra, and do not affect the findings here. The binding energy scale was calibrated by setting the peak position of measured $\mathrm{Au}_{4} \mathrm{f}_{7 / 2}$ peaks, recorded from a reference gold film, to 84.0 eV. Curve-fitting was performed with XPSPEAK 4.1. Quantification was performed by deriving sensitivity factors from measurements of the precursor films, and assuming the atomic ratios (e.g. $\mathrm{I}: \mathrm{Pb}=2: 1$ for $\mathrm{PbI}_{2}$ ) to be correct.

\section{Supporting Information}

Supporting Information is available from the Wiley Online Library or from the author.

\section{Acknowledgements}

Z.A.-G. acknowledges funding from a Winton Studentship, and ICON Studentship from the Lloyd's Register Foundation. S.D.S acknowledge the European Research Council (ERC) under the European Union's Horizon 2020 research and innovation programme (HYPERION, Grant Agreement No. 756962), and the Royal Society and Tata Group (UF150033). The authors thank the Engineering and Physical Sciences Research Council (EPSRC) for support (EP/R023980/1). M.A.-J. thanks Cambridge Materials Limited, Wolfson College, University of Cambridge, and EPSRC for their funding and technical support. F.U.K. thanks the Jardine Foundation and Cambridge Trust for a doctoral scholarship. This work was carried out with the support of the Diamond Light Source, instrument I09 (proposal SI22668-1). The research leading to this result has been supported by the project CALIPSOplus under Grant Agreement 730872 from the EU Framework Programme for Research and Innovation HORIZON 2020. H.R., G.J.M. and U.B.C. acknowledge research funding from the Swedish Research Council (Grant nos. VR 2018-04125, 2018-06465, and 2018-04330), the Swedish Foundation for Strategic Research (Project no. RMA15-0130) and the Swedish Energy Agency (Grant no. P43549-1).

Received: ((will be filled in by the editorial staff))

Revised: ((will be filled in by the editorial staff)) Published online: ((will be filled in by the editorial staff))

\section{References}

[1] W. S. Yang, B.-W. Park, E. H. Jung, N. J. Jeon, Y. C. Kim, D. U. Lee, S. S. Shin, J. Seo, E. K. Kim, J. H. Noh, S. Il Seok, Science 2017, 356, 1376.

[2] C. C. Stoumpos, C. D. Malliakas, M. G. Kanatzidis, Inorg. Chem. 2013, 52, 9019.

[3] S. D. Stranks, H. J. Snaith, Nat. Nanotechnol. 2015, 10, 391. 


\section{WILEY-VCH}

[4] J. H. Noh, S. H. Im, J. H. Heo, T. N. Mandal, S. Il Seok, Nano Lett. 2013, 13, 1764.

[5] L. K. Ono, E. J. Juarez-Perez, Y. Qi, ACS Appl. Mater. Interfaces 2017, 9, 30197.

[6] C. Yi, J. Luo, S. Meloni, A. Boziki, N. Ashari-Astani, C. Grätzel, S. M. Zakeeruddin,

U. Röthlisberger, M. Grätzel, Energy Environ. Sci. 2016, 9, 656.

[7] M. Ibrahim Dar, M. Abdi-Jalebi, N. Arora, T. Moehl, M. Grätzel, M. K. Nazeeruddin, Adv. Mater. 2015, 27, 7221.

[8] D. P. McMeekin, G. Sadoughi, W. Rehman, G. E. Eperon, M. Saliba, M. T. Hörantner, A. Haghighirad, N. Sakai, L. Korte, B. Rech, M. B. Johnston, L. M. Herz, H. J. Snaith, Science 2016, 351, 151.

[9] B. Zhao, S. Bai, V. Kim, R. Lamboll, R. Shivanna, F. Auras, J. M. Richter, L. Yang, L. Dai, M. Alsari, X.-J. She, L. Liang, J. Zhang, S. Lilliu, P. Gao, H. J. Snaith, J. Wang, N. C. Greenham, R. H. Friend, D. Di, Nat. Photonics 2018, 12, 783.

[10] Y. Cao, N. Wang, H. Tian, J. Guo, Y. Wei, H. Chen, Y. Miao, W. Zou, K. Pan, Y. He, H. Cao, Y. Ke, M. Xu, Y. Wang, M. Yang, K. Du, Z. Fu, D. Kong, D. Dai, Y. Jin, G. Li, H. Li, Q. Peng, J. Wang, W. Huang, Nature 2018, 562, 249.

[11] K. Lin, J. Xing, L. N. Quan, F. P. G. de Arquer, X. Gong, J. Lu, L. Xie, W. Zhao, D. Zhang, C. Yan, W. Li, X. Liu, Y. Lu, J. Kirman, E. H. Sargent, Q. Xiong, Z. Wei, Nature 2018, 562, 245.

[12] Y. Shen, L. Cheng, Y. Li, W. Li, J. Chen, S. Lee, J. Tang, Adv. Mater. 2019, 1901517.

[13] A. Sadhanala, A. Kumar, S. Pathak, A. Rao, U. Steiner, N. C. Greenham, H. J. Snaith, R. H. Friend, Adv. Electron. Mater. 2015, 1, 1500008.

[14] S. A. Veldhuis, P. P. Boix, N. Yantara, M. Li, T. C. Sum, N. Mathews, S. G. Mhaisalkar, Adv. Mater. 2016, 28, 6804.

[15] M. Anaya, B. P. Rand, R. J. Holmes, D. Credgington, H. J. Bolink, R. H. Friend, J. Wang, N. C. Greenham, S. D. Stranks, Nat. Photonics 2019, 13, 818.

[16] M. Yang, N. Wang, S. Zhang, W. Zou, Y. He, Y. Wei, M. Xu, J. Wang, W. Huang, J. 


\section{WILEY-VCH}

Phys. Chem. Lett. 2018, 9, 2038.

[17] Y. Ke, N. Wang, D. Kong, Y. Cao, Y. He, L. Zhu, Y. Wang, C. Xue, Q. Peng, F. Gao, W. Huang, J. Wang, J. Phys. Chem. Lett. 2019, 10, 380.

[18] M. K. Gangishetty, S. N. Sanders, D. N. Congreve, ACS Photonics 2019, 6, 1111.

[19] F. Jin, B. Zhao, B. Chu, H. Zhao, Z. Su, W. Li, F. Zhu, J. Mater. Chem. C 2018, 6, 1573.

[20] Q. Dong, F. Liu, M. K. Wong, H. W. Tam, A. B. Djurišić, A. Ng, C. Surya, W. K. Chan, A. M. C. Ng, ChemSusChem 2016, 9, 2597.

[21] Q. Dong, L. Lei, J. Mendes, F. So, J. Phys. Mater. 2020, 3, 012002.

[22] H. Cho, Y.-H. Kim, C. Wolf, H.-D. Lee, T.-W. Lee, Adv. Mater. 2018, 30, 1704587.

[23] L. Zhao, K. M. Lee, K. Roh, S. U. Z. Khan, B. P. Rand, Adv. Mater. 2019, 31, 1805836.

[24] Y. Luo, P. Khoram, S. Brittman, Z. Zhu, B. Lai, S. P. Ong, E. C. Garnett, D. P. Fenning, Adv. Mater. 2017, 29, 1703451.

[25] S. Bai, P. Da, C. Li, Z. Wang, Z. Yuan, F. Fu, M. Kawecki, X. Liu, N. Sakai, J. T.-W. Wang, S. Huettner, S. Buecheler, M. Fahlman, F. Gao, H. J. Snaith, Nature 2019, 571, 245.

[26] H. Kim, J. S. Kim, J. M. Heo, M. Pei, I. H. Park, Z. Liu, H. J. Yun, M. H. Park, S. H. Jeong, Y. H. Kim, J. W. Park, E. Oveisi, S. Nagane, A. Sadhanala, L. Zhang, J. J. Kweon, S. K. Lee, H. Yang, H. M. Jang, R. H. Friend, K. P. Loh, M. K. Nazeeruddin, N. G. Park, T. W. Lee, Nat. Commun. 2020, 11, 1.

[27] C.-C. Zhang, Z.-K. Wang, M. Li, Z.-Y. Liu, J.-E. Yang, Y.-G. Yang, X.-Y. Gao, H. Ma, J. Mater. Chem. A 2018, 6, 1161.

[28] Z. Andaji-Garmaroudi, M. Anaya, A. J. Pearson, S. D. Stranks, Adv. Energy Mater. 2020, 10, 1903109.

[29] M. Abdi-Jalebi, Z. Andaji-Garmaroudi, S. Cacovich, C. Stavrakas, B. Philippe, J. M. 


\section{WILEY-VCH}

Richter, M. Alsari, E. P. Booker, E. M. Hutter, A. J. Pearson, S. Lilliu, T. J. Savenije, H. Rensmo, G. Divitini, C. Ducati, R. H. Friend, S. D. Stranks, Nature 2018, 555, 497.

[30] T. A. S. Doherty, A. J. Winchester, S. Macpherson, D. N. Johnstone, V. Pareek, E. M. Tennyson, S. Kosar, F. U. Kosasih, M. Anaya, M. Abdi-Jalebi, Z. Andaji-Garmaroudi, E. L. Wong, J. Madéo, Y.-H. Chiang, J.-S. Park, Y.-K. Jung, C. E. Petoukhoff, G.

Divitini, M. K. L. Man, C. Ducati, A. Walsh, P. A. Midgley, K. M. Dani, S. D. Stranks, Nature 2020, 580, 360.

[31] M. C. Brennan, S. Draguta, P. V. Kamat, M. Kuno, ACS Energy Lett. 2018, 3, 204.

[32] H. Lee, D. Ko, C. Lee, ACS Appl. Mater. Interfaces 2019, 11, 11667.

[33] Z. Andaji-Garmaroudi, M. Abdi-Jalebi, D. Guo, S. Macpherson, A. Sadhanala, E. M. Tennyson, E. Ruggeri, M. Anaya, K. Galkowski, R. Shivanna, K. Lohmann, K. Frohna, S. Mackowski, T. J. Savenije, R. H. Friend, S. D. Stranks, Adv. Mater. 2019, 31, 1902374.

[34] N. Ahn, K. Kwak, M. S. Jang, H. Yoon, B. Y. Lee, J.-K. Lee, P. V. Pikhitsa, J. Byun, M. Choi, Nat. Commun. 2016, 7, 13422.

[35] S. Chen, X. Wen, R. Sheng, S. Huang, X. Deng, M. A. Green, A. Ho-Baillie, ACS Appl. Mater. Interfaces 2016, 8, 5351.

[36] M. Abdi-Jalebi, Z. Andaji-Garmaroudi, S. Cacovich, C. Stavrakas, B. Philippe, J. M. Richter, M. Alsari, E. P. Booker, E. M. Hutter, A. J. Pearson, S. Lilliu, T. J. Savenije, H. Rensmo, G. Divitini, C. Ducati, R. H. Friend, S. D. Stranks, Nature 2018, 555, 497.

[37] F. Zheng, W. Chen, T. Bu, K. P. Ghiggino, F. Huang, Y. Cheng, P. Tapping, T. W. Kee, B. Jia, X. Wen, Adv. Energy Mater. 2019, 9, 1901016.

[38] M. Abdi-Jalebi, Z. Andaji-Garmaroudi, A. J. Pearson, G. Divitini, S. Cacovich, B. Philippe, H. Rensmo, C. Ducati, R. H. Friend, S. D. Stranks, ACS Energy Lett. 2018, 3, 2671.

[39] L. Wang, D. Moghe, S. Hafezian, P. Chen, M. Young, M. Elinski, L. Martinu, S. Kéna- 


\section{WILEY-VCH}

Cohen, R. R. Lunt, ACS Appl. Mater. Interfaces 2016, 8, 23086.

[40] M. Hu, C. Bi, Y. Yuan, Y. Bai, J. Huang, Adv. Sci. 2016, 3, 1500301.

[41] N. Li, L. Song, Y. Jia, Y. Dong, F. Xie, L. Wang, S. Tao, N. Zhao, Adv. Mater. 2020, $32,1907786$.

[42] C. Cho, B. Zhao, G. D. Tainter, J.-Y. Lee, R. H. Friend, D. Di, F. Deschler, N. C. Greenham, Nat. Commun. 2020, 11, 611.

[43] J. C. De Mello, H. F. Wittmann, R. H. Friend, Adv. Mater. 1997, 9, 230.

[44] F. U. Kosasih, C. Ducati, Nano Energy 2018, 47, 243.

[45] S. Cacovich, F. Matteocci, M. Abdi-Jalebi, S. D. Stranks, A. Di Carlo, C. Ducati, G. Divitini, ACS Appl. Energy Mater. 2018, 1, 7174.

[46] S. Tanuma, C. J. Powell, D. R. Penn, Surf. Interface Anal. 2011, 43, 689. 